\title{
ON THE GENERALIZED SEIDEL CLASS $U$
}

BY

JUN SHUNG HWANG ${ }^{1}$

To the memory of my advisor Wladimir Seidel

\begin{abstract}
As usual, we say that a function $f \in U$ if $f$ is meromorphic in $|z|<1$ and has radial limits of modulus 1 a.e. (almost everywhere) on an $\operatorname{arc} A$ of $|z|=1$. This paper contains three main results: First, we extend our solution of A. J. Lohwater's problem (1953) by showing that if $f \in U$ and $f$ has a singular point $P$ on $A$, and if $v$ and $1 / \bar{v}$ are a pair of values which are not in the range of $f$ at $P$, then one of them is an asymptotic value of $f$ at some point of $A$ near $P$. Second, we extend our solution of J. L. Doob's problem (1935) from analytic functions to meromorphic functions, namely, if $f \in U$ and $f(0)=0$, then the range of $f$ over $|z|<1$ covers the interior of some circle of a precise radius depending only on the length of $A$. Finally, we introduce another class of functions. Each function in this class has radial limits lying on a finite number of rays a.e. on $|z|=1$, and preserves a sector between domain and range. We study the boundary behaviour and the representation of functions in this class.
\end{abstract}

1. Introduction. Let $D=\{z:|z|<1\}, C=\{z:|z|=1\}$, and $A(\alpha, \beta)=\left\{e^{i \theta}\right.$ : $\alpha<\theta<\beta\}$. Following E. F. Collingwood and A. J. Lohwater [1, p. 107], we let $U$ be the Seidel class containing all nonconstant functions $f(z)$ meromorphic in $D$ for which the radial limits $f\left(e^{i \theta}\right)$ exist and have modulus 1 for almost all points $e^{i \theta}$ on $C$. In particular, if the above properties hold on an $\operatorname{arc} A(\alpha, \beta)$ of $C$ then we say that $f \in U$ on $A(\alpha, \beta)$. The name of this class is for Seidel due to his contributions [11, 12].

In [7, Theorem 3], Lohwater proved that if $f \in U$ on $A(\alpha, \beta)$ with bounded characteristic in the sense of Nevanlinna (see [1, p. 38]), and if $P$ is a singular point of $f$ on $A(\alpha, \beta)$, then every value of modulus 1 which is not in the range of $f$ at $P$ is an asymptotic value of $f$ at some point of each subarc of $A(\alpha, \beta)$ containing the point $P$. He then asked whether this result is still true if $f$ is not of bounded characteristic (see [7, p. 156]). Recently, in [5], we have solved this problem in the affirmative sense as follows.

THEOREM 1. If $f \in U$ on $A(\alpha, \beta)$ and if $P$ is a singular point of $f$ on $A(\alpha, \beta)$, then every value of modulus 1 which is not in the range of $f$ at $P$ is an asymptotic value of $f$ at some point of each subarc of $A(\alpha, \beta)$ containing the point $P$.

Received by the editors September 15, 1981 and, in revised form, March 10, 1982.

1980 Mathematics Subject Classification. Primary 30C80; Secondary 30C45, 30C50.

Key words and phrases. Boundary behaviour, generalized Seidel class, reflection principle, sector preserving function, and star-like function.

'I am indebted to the referee for his many valuable comments and corrections in this paper. 
Note that in our more recent paper [6, Theorem 1], we gave an alternate proof of Theorem 1 via the very method of Lohwater which was apparently thought to be impossible by him [7, p. 156].

In view of the hypothesis of Theorem 1, we may naturally ask whether the restriction "every value of modulus 1 " is necessary. The answer turns out to be yes; for instance, the function $f(z)=e^{-(1+z) /(1-z)}$ belongs to the class $U$, but any value $v$ with $|v|>1$ is not in the range of $f$ at $P=1$ and clearly is not an asymptotic value of $f$.

Regarding the extension of Theorem 1, we may therefore ask whether the restriction "every value of modulus 1 " can be improved by the hypothesis "every pair of values $v$ and $1 / \bar{v}$ ". This gives us the motivation to formulate the following extension of Theorem 1 .

TheORem 2. Let $f \in U$ on $A(\alpha, \beta)$ and let $P$ be a singular point of $f$ on $A(\alpha, \beta)$. If $v$ and $1 / \bar{v}$ are a pair of values which are not in the range of $f$ at $P$, then one of them is an asymptotic value of $f$ at some point of each subarc of $A(\alpha, \beta)$ containing the point $P$.

Here we make two remarks: First, if $v$ is a value of modulus 1 , then clearly we have $v=1 / \bar{v}$, so that Theorem 1 follows from Theorem 2. Second, the first two methods introduced in $[5,6]$ cannot give Theorem 2 . To see this, we need only observe the key transformations introduced in [5, 6], namely, $g(z)=1 /(f(z)-v)$ and $h(z)=\exp \{(f(z)+v) /(f(z)-v)\}$. Both of them involve only the value $v$. Therefore, to prove Theorem 2, we shall need the following Moebius transformation which involves both $v$ and $1 / \bar{v}$,

$$
m_{f}(z)=(f(z)-v) /(1-\bar{v} f(z)) .
$$

2. Invariance of Seidel's class. Before proving Theorem 2, we shall first formulate the following invariance of class $U$ under the Moebius transformation.

LEMma 1. Let $v$ be a value with $|v| \neq 1$. Then $f \in U$ if and only if $m_{f} \in U$, where $m_{f}$ is defined by (1).

3. Extension of the Schwarz reflection principle. To prove Theorem 2, we shall need the following extension of the reflection principle.

LEMMA 2. If $f \in U$ on $A(\alpha, \beta)$ and if $f$ is both bounded above and below in a vicinity of $A(\alpha, \beta)$ relative to $D$, then $f$ may be continued analytically across the arc $A(\alpha, \beta)$ by means of the reflection principle

$$
f(1 / z)=1 / \bar{f}(\bar{z})
$$

Clearly, the proof of Lemma 2 will be the same as that of Collingwood and Lohwater [1, p. 94] and therefore we omit the details.

As an application of Lemma 2, we shall prove the following theorem of Lohwater [7, Theorem 2] which will be needed in proving Theorem 2.

LEMMA 3. If $f \in U$ on $A(\alpha, \beta)$ and if $f$ has no zeros and poles in a vicinity of $A(\alpha, \beta)$ relative to $D$, then a necessary and sufficient condition that $f$ can be continued 
analytically beyond $A(\alpha, \beta)$ is that $f$ admits neither 0 nor $\infty$ as asymptotic values on $A(\alpha, \beta)$.

Proof. The necessity is obvious, because if $f$ is analytic beyond $A(\alpha, \beta)$ then $f$ is continuous there, so that $f$ admits neither 0 nor $\infty$ as asymptotic values on $A(\alpha, \beta)$.

Conversely, if $f$ has a singular point $P \in A(\alpha, \beta)$, then by Lemma $2 f$ must be either unbounded above or below. In either case, by applying the constructive method introduced in [5] or [6, Theorem 4], there can be constructed a path $\Gamma$ ending at a point $P^{\prime}$ on $A(\alpha, \beta)$ arbitrarily close to $P$ such that $f$ tends to either $\infty$ or 0 along $\Gamma$ depending on either $f$ being unbounded above or below, respectively. This completes the proof.

Here we would like to make two remarks. First, we point out that a clarification is needed in the aforementioned theorem of Lohwater. To see this, let us review the hypothesis of Lemma 3. We require that the function $f$ have no zeros and poles in a vicinity of $A(\alpha, \beta)$ while Lohwater stated in his theorem that $f$ has no zeros or poles in such a vicinity. In view of his proof, we can see that he did require the function $f$ to be free from both zeros and poles. We want to emphasize that the assertion of Lohwater's theorem will be false if the function $f$ has merely no zeros in a vicinity of $A(\alpha, \beta)$. For instance, the following reciprocal of the Weierstrass product (see Seidel $[12$, p. 214]),

$$
w(z)=\left(1-e^{-1} \exp \left(\frac{z+1}{z-1}\right)\right) /\left(\exp \left(\frac{z+1}{z-1}\right)-e^{-1}\right),
$$

has no zeros in $D$ and admits neither 0 nor $\infty$ as asymptotic values on $C$. Clearly, this function $w \in U$ and it cannot be continued analytically beyond the whole circle $C$ because of the singularity $z=1$. Furthermore, $w$ has a sequence of poles tending to 1 . This shows that the function $f$ in Lemma 3 must be free from both zeros and poles in a vicinity of $A(\alpha, \beta)$.

Second, we remark that the assertion of Lemma 3 can be extended to a more general class. As in [6], we let $|U|$ be the class of all meromorphic functions $f$ such that the radial limits of the modulus $\lim _{r \rightarrow 1}\left|f\left(r e^{i \theta}\right)\right|=1$ a.e. on $C$ or an arc of $C$. In this case, a function $f$ in the class $|U|$ may have no radial limits (see [6, Theorem 2]). However, the related theorem in [6, Theorem 3] is still true for functions in $|U|$, so that Lemma 3 can be extended to this class. Unfortunately, whether Theorem 2 itself can be extended to this class, in general, we do not know. Of course, if $|v| \neq 1$, then such an extension is true (see also [6, Theorem 4]).

4. Proof of Theorem 2. With the help of Theorem 1 and Lemmas 1 and 3, we can now easily prove Theorem 2 . Let $v$ and $1 / \bar{v}$ be a pair of values which are not in the range of $f$ at a singular point $P \in A(\alpha, \beta)$. If $v$ is a value of modulus 1 , then the assertion follows from Theorem 1. We may therefore assume that $|v| \neq 1$. Since $f \in U$ on $A(\alpha, \beta)$, it follows from Lemma 1 that the Moebius transformation $m_{f} \in U$ on $A(\alpha, \beta)$. Clearly, the function $m_{f}$ has no zeros and poles in a vicinity of $A(\alpha, \beta)$ relative to $D$. Furthermore, the point $P$ is also a singularity of $m_{f}$ and therefore by applying Lemma 3 we conclude that the function $m_{f}$ admits either 0 or $\infty$ as asymptotic values at a point $P^{\prime}$ on $C$ arbitrarily close to $P$. This in turn implies 
that either $v$ or $1 / \bar{v}$ is an asymptotic value of the function $f$ at the point $P^{\prime}$ and the proof is complete.

5. Doob's class. Following Doob [2, p. 119], we say that a function $f(z)$ analytic in $D$ has the property $K(\rho)$ if $f(0)=0$ and for some $\operatorname{arc} A(\alpha, \alpha+2 \rho), \rho>0$, we have

$$
\lim \inf \left|f\left(P_{i}\right)\right| \geqslant 1 \text {, }
$$

where $\left\{P_{i}\right\}$ is a sequence of points in $D$ tending to a point on $A(\alpha, \alpha+2 \rho)$.

Instead of analytic functions, we may consider meromorphic functions and we denote by $K^{*}(\rho)$ the same property for meromorphic functions. In view of the definition (3), we can see that any function $f$, with $f(0)=0$, in the generalized Seidel class is also a function in the generalized Doob class.

Recently, in [3, Theorem 5], we have answered an open problem of Doob [2, p. 120] by showing that

THEOREM 3. If $f \in K(\rho)$, then the range of $f$ covers the interior of some circle of radius

$$
k(\rho)=1 / 16 N(\rho), \quad N(\rho)=\max \left(\pi e^{\sqrt{2}} / 2, e \log 1 /(1-\cos \rho)\right) .
$$

We take this time to make up a remark as follows: The number $k(\rho)$ in [3] is written as $k(\rho)=1 /(16 e \log 1 /(1-\cos \rho))$, which is true only for small $\rho$, say $\rho<\pi / 6$. In general, the number $k(\rho)$ should be (4). Of course, the estimate in (4) is not best possible. The best one is still open to us. What we can do here is to extend the above to the case of meromorphic functions as follows.

THEOREM 4. If $f \in K^{*}(\rho)$, then the assertion of Theorem 3 is still true.

In fact, Theorem 4 is an immediate consequence of Theorem 3 and the following covering property of meromorphic functions which will be needed in the next section.

LEMMA 4. Let $f$ be meromorphic in $D$ and have a pole at $p \in D$. If $f$ has no zeros in the closed disk $\bar{D}_{r}(p)=\{z:|z-p| \leqslant r\}$, where $0<r<1-|p|$, and if $M=$ $\max |f(z)|$ for $|z|=r$, then the range

$$
f\left(D_{r}(p)\right) \supset\{w:|w|>M\} .
$$

Proof. Let $g(z)=1 / f(z)$. Then by the hypotheses the function $g$ has a zero at $p$, is analytic in $\bar{D}_{r}(p)$, and further satisfies

$$
\min _{|z|=r}|g(z)|=1 / M, \quad \text { where } M=\max _{|z|=r}|f(z)| .
$$

Let $w_{0}$ be an arbitrary point in the set $\{w:|w|>M\}$ and let $h(z)=g(z)-1 / w_{0}$, where $\left|1 / w_{0}\right|<1 / M$. Then we have

$$
|h(z)-g(z)|=\left|1 / w_{0}\right|<|g(z)|, \text { for all points on }|z|=r \text {. }
$$

Since $g$ has a zero in $D_{r}(p)$, it follows from Rouchés theorem (see [8, Theorem 10.10]) that the function $h$ has a zero $z_{0}$ in $D_{r}(p)$. This yields that $f\left(z_{0}\right)=w_{0}$ and the proof is complete. 
From the above lemma we see that if $f$ has a pole in $D$ then the range of $f$ covers the interior of some circle of arbitrarily large radius. This, together with Theorem 3 , yields Theorem 4.

Note that in [4, p. 625], we have introduced a generalized Doob class $K(r, \rho, s)$ for analytic functions. As far as the covering property is concerned, the related theorem [4, Theorem 4] is also true if we allow the associated functions to be meromorphic.

6. Sector preserving functions. Finally, we shall introduce a class of functions which preserve sectors. For this, we let

$$
\Delta(\alpha, \beta)=\{z: 0<|z|<\infty \text { and } \alpha<\arg z<\beta\}, \quad \beta-\alpha<2 \pi,
$$

be a sector and let $R_{1}, R_{2}, \ldots, R_{n}$ be $n$ distinct rays passing through the origin. Denote by $\Delta(\alpha, \beta ; n)$ the class of all functions $f(z)$ meromorphic in $D$ such that the radial limits

$$
f\left(e^{i \theta}\right) \in R_{j}, \quad \text { a.e. on }\left[\alpha_{j}, \alpha_{j+1}\right] \text {, }
$$

where $j=1,2, \ldots, n$ and $\alpha_{1}<\alpha_{2}<\cdots<\alpha_{n+1}=\alpha_{1}+2 \pi$, and the range $f(D)$ preserves the sector $\Delta(\alpha, \beta)$ in the following sense:

$$
f(D \cap \Delta(\alpha, \beta)) \subset \Delta(\alpha, \beta) \text { and } f\left(D \cap \Delta(\alpha, \beta)^{c}\right) \subset \Delta(\alpha, \beta)^{c} \text {, }
$$

where $\Delta(\alpha, \beta)^{c}$ denotes the complement of $\Delta(\alpha, \beta)$. Functions of this kind will be referred as sector preserving functions. Such functions do exist, for instance, the function (refer to Corollary 2)

$$
f_{n}(z)=z /\left(1-z^{n}\right)^{2 / n}, \quad \text { where } n \text { is an integer, }
$$

maps $D$ conformally onto the complement of the $n$ half rays

$$
R_{j}^{*}=\left\{w: 2^{-2 / n}<|w|<\infty \text { and } \arg w=(2 j-1) \pi / n\right\},
$$

and preserves the $2 n$ sectors

$$
\Delta_{k}=\{w: 0<|w|<\infty \text { and } k \pi / n<\arg w<(k+1) \pi / n\},
$$

where $j=1,2, \ldots, n$, and $k=0, \ldots, 2 n-1$.

Note that the class of sector preserving functions can be considered as a special subclass of the generalized Seidel class $U$. To see this, we let $f \in \Delta(\alpha, \beta ; n)$ and let $H_{j}$ be one of the half-planes separated by the ray $R_{j}$. Denote by $w_{j}$ a conformal mapping from $H_{j}$ onto $D$. Then by (5), we can see that the function $w_{j} \circ f$ has radial limits of modulus 1 , a.e. on $A\left(\alpha_{j}, \alpha_{j+1}\right)$, so that $w_{j} \circ f \in U$.

Also note that, as usual (see Chr. Pommerenke [9, p. 42]), a function $f$ is called star-like in $D$ if it is univalent in $D$ and its range $f(D)$ is a domain star-like with respect to the origin.

Having the above two remarks, we shall now state the following criterion of sector preserving functions.

THEOREM 5. If $f \in \Delta(\alpha, \beta ; n)$ with $\beta-\alpha \neq \pi$, and if $f(0) \neq \infty$, then $f$ has no poles and zeros in $D$ except for a simple zero at the origin, and no essential singularities on $C$. Furthermore, if $f$ is univalent in $D$ then $f$ has exactly $n$ algebraic poles at $e^{i \alpha_{j}}$, 
$j=1,2, \ldots, n$, no other poles or branch points on $C, f$ is star-like in $D$ and can be precisely represented by

$$
f(z)=c z \prod_{j=1}^{n}\left(1-e^{-i \alpha_{j}} z\right)^{-p_{j}},
$$

where $c=f^{\prime}(0) \neq 0, \sum_{j=1}^{n} p_{j}=2$, and $p_{j} \pi$ denotes the angle between $R_{j}$ and $R_{j+1}$.

Note that from the definition of Seidel's class we can see that a function $f \in U$ if and only if $1 / f \in U$. The same property holds for functions in the class $\Delta(\alpha,-\alpha ; n)$ due to the above theorem. This reciprocal relation explains the reason why the normalization $f(0) \neq \infty$ is necessary in order to prove that $f$ has a simple zero at the origin. Furthermore, the hypotheses that $\beta-\alpha \neq \pi$ and $f$ be univalent are both necessary in proving the representation (8). Two counterexamples are provided in the last section.

7. Preliminary lemmas. The proof of Theorem 5 is lengthy, and for convenience we separate the details into the following seven lemmas. We begin with proving that $f$ has no poles in a subdomain of $D$.

LEMMA 5. If $f \in \Delta(\alpha, \beta ; n)$ and if $\partial \Delta(\alpha, \beta)$ denotes the boundary of the sector $\Delta(\alpha, \beta)$, then $f$ has no poles in $D-\partial \Delta(\alpha, \beta)$.

Proof. Suppose on the contrary that $f$ has a pole $p$ in $D-\partial \Delta(\alpha, \beta)$. We may, without loss of generality, assume that $p \in \Delta(\alpha, \beta)$ because the other case can be proved by the same argument. Since $f$ is meromorphic in $D$, there can be chosen a positive number $r<1-|p|$ such that $f$ has no poles and zeros in the set $D_{r}(p)=\{z$ : $0<|z-p| \leqslant r\}$ and $D_{r}(p) \subset \Delta(\alpha, \beta)$. It then follows from Lemma 4 that the range

$$
f\left(D_{r}(p)\right) \supset\{w:|w|>M\}, \quad \text { where } M=\max _{|z|=r}|f(z)| .
$$

Since $D_{r}(p) \subset \Delta(\alpha, \beta)$, the above inclusion violates the first relation of (6). This proves the lemma.

Before extending Lemma 5, we shall first prove the following invariance of the boundary $\partial \Delta(\alpha, \beta)$ under a mapping in the class $\Delta(\alpha, \beta ; n)$.

Lemma 6. If $f \in \Delta(\alpha, \beta ; n)$, and if $z \in \partial \Delta(\alpha, \beta)$ which is not a pole of $f$, then the image $f(z) \in \partial \Delta(\alpha, \beta)$.

Proof. Suppose on the contrary that $f(z) \notin \partial \Delta(\alpha, \beta)$. We may, without loss of generality, assume that $f(z) \in \Delta(\alpha, \beta)$. Then by the continuity of $f$, there is a vicinity $V$ of $z$ such that the image $f(V) \subset \Delta(\alpha, \beta)$, where $V \subset D$. Since $z \in \partial \Delta(\alpha, \beta)$, so that $V \cap \Delta(\alpha, \beta)^{c} \neq \varnothing$. Thus the above inclusion violates the second relation of (6) and the lemma is proved.

With the help of Lemma 6, we are now able to prove the following extension of Lemma 5.

LemMa 7. If $f \in \Delta(\alpha, \beta ; n)$ with $\beta-\alpha \neq \pi$, and if $f(0) \neq \infty$, then $f$ has no poles in D. 
Proof. We shall prove that $f$ has no poles on $\partial \Delta(\alpha, \beta) \cap D$. Suppose on the contrary that $f$ has a pole of order $k$ at a point $p=r e^{i \alpha}, r>0$. Then by the Laurent expansion, $f$ can be written as

$$
f(z)=\left(c_{-k}+g(z)\right) /(z-p)^{k} \quad\left(c_{-k} \neq 0, k>0\right),
$$

where the function $g$ is analytic at $p$ and $g(p)=0$. Let $c_{-k}=s e^{i a}$ and $z=\rho e^{i \alpha}$. Then we have

$$
f\left(\rho e^{i \alpha}\right)=s(\rho-r)^{-k} e^{i(a-k \alpha)}(1+o(\rho)),
$$

where the function $o(\rho) \rightarrow 0$, as $\rho \rightarrow r$, due to $g(p)=0$. Since $f(0) \neq \infty$, it follows from Lemma 6 that

$$
\arg f\left(\rho e^{i \alpha}\right)=\alpha \text { or } \beta, \text { for all } 0<\rho<1 \text { and } \rho \neq r .
$$

Letting $\rho$ tend to $r$ from above and below, we obtain the following four possible cases:

$$
a-k \alpha=\theta_{1} \text { and } a-k(\alpha+\pi)=\theta_{2},
$$

where $\left(\theta_{1}, \theta_{2}\right)=(\alpha, \alpha),(\alpha, \beta),(\beta, \alpha)$, or $(\beta, \beta)$. Clearly, with respect to each case in order, we get $k \pi=\theta_{1}-\theta_{2}=0, \alpha-\beta, \beta-\alpha$, or 0 , which is absurd due to $k>0$, $0<\beta-\alpha<2 \pi$, and the hypothesis $\beta-\alpha \neq \pi$. This yields that $f$ has no poles on $\partial \Delta(\alpha, \beta) \cap D$. Combining with Lemma 5, we conclude that $f$ has no poles in $D$. This proves the lemma.

Based on the above lemmas, we shall prove that the mapping $f$ is conformal at the origin and has no zeros in $D$ other than the origin.

LEMMA 8. Under the hypothesis of Lemma 7, the function $f$ has one and only one simple zero at the origin.

Proof. We shall first prove that $f$ has a simple zero at the origin. To the contrary we suppose that $f(0) \neq 0$. Since $f(0) \neq \infty$, it follows from Lemma 6 that the point $f(0)$ must lie on $\partial \Delta(\alpha, \beta)$. Applying the Taylor expansion

$$
f(z)=f(0)+a_{k} z^{k}+\ldots, \quad \text { where } k \geqslant 1,
$$

we see that the local property of $f$ at the origin is similar to that of $z^{k}$. It follows that the angles $\beta-\alpha$ and $2 \pi-(\beta-\alpha)$ of $\Delta(\alpha, \beta)$ and $\Delta(\alpha, \beta)^{c}$, respectively, are mapped by $f$ onto the angles $k(\beta-\alpha)$ and $k(2 \pi-(\beta-\alpha))$. Since $f(0) \neq 0$ and $f(0) \in \partial \Delta(\alpha, \beta)$, it follows from Lemma 6 that both angles must equal $\pi$, that is $k(\beta-\alpha)=k(2 \pi-(\beta-\alpha))=\pi$.This yields that $k=1$ and $\beta-\alpha=\pi$, contradicting the hypothesis $\beta-\alpha \neq \pi$. Hence we obtain $f(0)=0$. Substituting $f(0)=0$ into (9), we find that $k(\beta-\alpha)=\beta-\alpha$ or $k=1$, so that $f^{\prime}(0) \neq 0$. This proves that $f$ has a simple zero at the origin.

It remains to show that $f$ has no zeros in $D$ other than the origin. Suppose on the contrary that $f(q)=0$ for some $q \in D$ and $q \neq 0$. We then have two cases to consider: either $q \notin \partial \Delta(\alpha, \beta)$ or not. In the first case, we may assume that $q \in$ $\Delta(\alpha, \beta)$. Then by Rouche's theorem and the argument used in Lemma 4 , there are two disks

$$
D_{r}(q)=\{z:|z-q|<r\} \subset \Delta(\alpha, \beta) \text { and } D_{s}=\{w:|w|<s\}
$$


such that the range $f\left(D_{r}(q)\right) \supset D_{s}$. This, however, contradicts the first relation of (6) due to $D_{s} \cap \Delta(\alpha, \beta)^{c} \neq \varnothing$.

As for the second case, by expanding $f(z)$ as a Taylor series around the point $q$, considering the local property of $f$ at $q$, and using Lemma 6 , we see that the angles $\pi$ on both sides of $q$ are mapped by $f$ onto the angles $(\beta-\alpha) / k$ and $(2 \pi-(\beta-\alpha)) / k$, respectively. Since the mapping $f(z)$ is locally similar to that of $(z-q)^{k}$, it follows that $(\beta-\alpha)=2 \pi-(\beta-\alpha)$ or $\beta-\alpha=\pi$, contradicting the hypothesis. This yields the desired result and the proof is complete.

Note that the condition $\beta-\alpha \neq \pi$ is necessary in the above lemma. If $\beta-\alpha=\pi$, then there does exist a function $f(z)$ in the class $\Delta(\alpha, \beta ; 1)$ for which $f(0) \neq 0$. To find such an example, we need only observe the following typically real function due to W. Rogosinski [10], $T(z)=z /(1-z)^{2}, z \in D$, which maps the upper and lower half disk of $D$ onto the upper and lower half plane, respectively. Then the function $f(z)=a+T(z)$, where $0<a<1 / 4$, has radial limits on the half ray $R_{a}^{-}=\{z$ : $-\infty<z<a-1 / 4\}$ and preserves the sector $\Delta(0, \pi)$, so that $f \in \Delta(0, \pi ; 1)$, where $f(0) \neq 0$.

We shall now apply Theorem 1 to prove that functions in the class $\Delta(\alpha, \beta ; n)$ are free from essential singularities on $C$.

LEMMA 9. Under the hypothesis of Lemma 7, the function $f$ has no essential singularities on $C$.

Proof. Suppose to the contrary that $f$ has an essential singularity $P$ on $C$. We may assume that $P \in \bar{\Delta}(\alpha, \beta)$ and $\Delta(\alpha, \beta)$ contains the positive real axis and is disjoint from the negative one. We write $P=e^{i \phi}$. Then by (5) the radial limits $f\left(e^{i \theta}\right)$ satisfy

$$
f\left(e^{i \theta}\right) \in R^{+}, \text {a.e. on }\left[\alpha_{j}, \alpha_{j+1}\right] \text {, }
$$

where $R^{+}=R_{j}$ is a ray contained in $\Delta(\alpha, \beta)$ and the point $\phi \in\left[\alpha_{j}, \alpha_{j+1}\right]$. Since $\Delta(\alpha, \beta)$ contains the positive real axis, by applying a rotation to the function $f$, we may, without loss of generality, assume that the ray $R^{+}$is the positive real axis. Let $R^{-}$be the negative real axis. Then $R^{-}$is disjoint from $\Delta(\alpha, \beta)$ due to the previous assumption made. In view of Lemma 8 , we know that the function $f$ is conformal at the origin, so there is a small arc $\Gamma$ ending at the origin and contained in $\Delta(\alpha, \beta)^{c}$ such that the range

$$
f(v) \in R^{-}, \quad \text { for each } v \in \Gamma, v \neq 0 .
$$

We shall reach a contradiction from (10), (11), and Theorem 1. For this, we let $w(z)$ be a conformal mapping from the upper half-plane onto the unit disk $D_{w}=\{w:|w|<1\}$. Since the ray $R^{+}$is the positive real axis, it follows from (10) that the composite function $w \circ f$ has radial limits $(w \circ f)\left(e^{i \theta}\right)$ of modulus 1 , a.e. on $\left[\alpha_{j}, \alpha_{j+1}\right]$, so that $w \circ f \in U$ on $A\left(\alpha_{j}, \alpha_{j+1}\right)$.

In view of (11), we see that for each $v \in \Gamma$ and $v \neq 0$ the value $V=(w \circ f)(v)$ has modulus one and is not in the range of the point $P$ due to the fact that $P \in \bar{\Delta}(\alpha, \beta)$ and $\Delta(\alpha, \beta) \cap R^{-}=\varnothing$. Since $P$ is also a singular point of $w \circ f$, it follows from Theorem 1 that the value $V$ is an asymptotic value of $w \circ f$ at some point $P^{\prime}$ on the $\operatorname{arc} A(\alpha, \beta)$ arbitrarily close to $P$. This is true whether or not the 
point $P=e^{i \alpha}$ or $e^{i \beta}$. We then have a path $\gamma$ lying in $\Delta(\alpha, \beta) \cap D$ and ending at $P^{\prime}$ such that $\lim _{z \rightarrow P^{\prime}}(w \circ f)(z)=V$, where $z \in \gamma$. This together with (11) implies that

$$
\lim _{z \rightarrow P^{\prime}} f(z)=w^{-1}(V)=f(v) \in R^{-},
$$

where $z \in \gamma$ and $w^{-1}$ is the inverse of $w$. Since $P^{\prime} \in \Delta(\alpha, \beta)$ and $R^{-} \subset \Delta(\alpha, \beta)^{c}$, the above relation contradicts the first inclusion of (6). We thus conclude that $f$ has no essential singularities on $C$.

Note that the function $f$ may have some algebraic poles on $C$; see the example in (7). If $P$ is an algebraic pole on the $\operatorname{arc} A\left(\alpha_{j}, \alpha_{j+1}\right)$, then the composite function $w \circ f$ is no longer in the class $U$ on $A\left(\alpha_{j}, \alpha_{j+1}\right)$, so that the argument in the above lemma does not exclude the existence of poles on $C$.

Also note that the hypotheses $\beta-\alpha \neq \pi$ and $f(0) \neq \infty$ imposed in the above lemma can actually be omitted. To see this, we first observe from Lemmas 7 and 8 that if a sector $\Delta(\alpha, \beta)$ is symmetric with respect to the real axis, then a function $f \in \Delta(\alpha, \beta ; n)$ if and only if $1 / f \in \Delta(\alpha, \beta ; n)$. This, together with the fact that a function $f$ has an essential singularity if and only if $1 / f$ does, allows us to assume that $f(0) \neq \infty$. Next, the hypothesis $\beta-\alpha \neq \pi$ is to ensure that $f(0)=0$. If $f(0) \neq 0$, we may consider the translation $g(x)=f(z)-f(0)$. In this case, the function $g$ may not be in the class $\Delta(\alpha, \beta ; n)$, because the radial limits $g\left(e^{i \theta}\right)$ can lie on $n$ lines instead of $n$ rays. However, with a suitable change, the above argument can yield the assertion without any difficulty.

Based on some of the above lemmas, we are now able to prove the following boundary behaviour of functions in the class $\Delta(\alpha, \beta ; n)$ which are univalent in $D$.

LEMMA 10. Under the hypothesis of Lemma 7, if, in addition, the function $f$ is univalent in $D$, then $f$ has exactly $n$ algebraic poles at $e^{i \alpha_{j}}, j=1,2, \ldots, n$, but no other poles or branch points on $C$, and further $f$ is star-like in $D$.

Proof. According to Lemma 9, we know that the function $f$ has no essential singularities on $C$. It follows that $f$ has at most finitely many poles or algebraic poles or branch points on $C$, and further, $f$ may be continued analytically across any arc between two consecutive algebraic singularities by means of the reflection principle similar to that of Lemma 2. Denote by $F$ the resulting extension function of $f$. Then $F=f$ on $D$, but $F(z)$ is now the value symmetric to $f(1 / \bar{z})$ with respect to some ray $R_{j}$ for $|z|>1$. Clearly, the function $F$ is continuous through the plane except at the set of poles. Since $F$ is univalent in $D$ and $F(0)=0$ by Lemma 8 , it follows from Rouche's theorem that the boundary values $F\left(e^{i \theta}\right) \neq 0$ for all $\theta \in[0,2 \pi]$, so that the minimum of $|F|$ over each interval $\left[\alpha_{j}, \alpha_{j+1}\right]$ is positive, $j=1,2, \ldots, n$. Let the minimum occur at $e^{i \beta_{j}}, \alpha_{j} \leqslant \beta_{j} \leqslant \alpha_{j+1}$, and let $R_{j}^{*}$ be the half-ray on $R_{j}$ issuing from the minimum point $F\left(e^{i \beta_{j}}\right)$ to the point at infinity. Then by (5) and the continuity of $F$, we have

$$
R_{j}^{*}=\left\{F\left(e^{i \theta}\right): \alpha_{j} \leqslant \theta \leqslant \alpha_{j+1}\right\}, \quad j=1,2, \ldots, n,
$$

where $R_{j}^{*}$ contains the point at infinity. 
We shall show that $F$ has no poles or algebraic singularities in the interior of $A\left(\alpha_{j}, \alpha_{j+1}\right)$. Suppose on the contrary that $F$ has a singularity at $e^{i \phi}, \alpha_{j}<\phi<\alpha_{j+1}$. It follows from the same argument as in Lemma 7 that if $e^{i \phi}$ is a pole, then $F$ fails to preserve a sector, a contradiction. Neither is $e^{i \phi}$ a branch point of $F$ due to the univalency of $F$ in $D$. Suppose now $e^{i \phi}$ is an algebraic pole. Then again by the univalency of $F$ in $D$, we see that the range $F\left(e^{i \theta}\right) \in R_{j}$ for $\alpha_{j}<\theta<\phi$ and $F\left(e^{i \theta}\right) \in R_{j+1}$ for $\phi<\theta<\alpha_{j+1}$. This together with (12) yields that $R_{j}=R_{j+1}$, violating the distinction of $R_{j}, j=1,2, \ldots, n$.

It remains to prove that both endpoints $e^{i \alpha_{j}}$ and $e^{i \alpha_{j+1}}$ are algebraic poles of $F$. This, however, is easy, because in the opposite case we must have either $R_{j}=R_{j-1}$ or $R_{j}=R_{j+1}$, a contradiction. We thus conclude that $F$ has exactly $n$ algebraic poles at $e^{i \alpha_{j}}$ and no other singularities on $C$.

Finally, we shall prove that the range $F(D)$ is star-like with respect to the origin. In view of (12), we know that the set of all boundary values

$$
B=\bigcup_{\theta=0}^{2 \pi} F\left(e^{i \theta}\right)=\bigcup_{j=1}^{n} R_{j}^{*} \text { and } 0 \notin B,
$$

where the endpoint of $R_{j}^{*}$ occurs at $F\left(e^{i \beta_{j}}\right)$ defined before (12). It follows that the complement $S=B^{c}$ is a star-like domain with respect to the origin. The assertion will be proved if we can show that the range $F(D)=S$.

We now prove that $S \subset F(D)$. Suppose this inclusion is false. Then there is a point $p$ such that $p \in S$ and $p \in \partial F(D)$. It follows that $p$ is an asymptotic value of $F$ in $D$, so that $p=F\left(e^{i \theta}\right)$ for some $\theta$, due to the continuity of $F$. This yields that $p \in B$, a contradiction, and the inclusion $S \subset F(D)$ is proved.

Conversely, we shall prove that $F(D) \subset S$. Suppose not; then there is a point $p \in D$ such that $F(p) \in B$. It follows from (13) that $F(p)=F\left(e^{i \theta}\right)$ for some $\theta$. Applying Rouché's theorem, we see that $F$ is at least two-valent in $D$ coming from two vicinities of $p$ and $e^{i \theta}$, a contradiction. This gives $F(D) \subset S$, so that $F(D)=S$. Hence the function $F$ is star-like in $D$. This proves the lemma.

As long as the star-like property of $f$ is proved, the representation of $f$ can be settled by a known result (see Pommerenke [9, Theorem 2.6]) as follows.

LEMMA 11. A function $f(z)=c z+\cdots$, is star-like in $D$ if and only if

$$
f(z)=c z \exp \left\{2 \int_{0}^{2 \pi} \log \frac{1}{1-e^{-i t z}} d \gamma(t)\right\},
$$

for some increasing function $\gamma(t)$ with $\gamma(2 \pi)-\gamma(0)=1$.

8. Proof of Theorem 5. With the help of Lemmas 7-11, we are now able to prove Theorem 5. Let $f \in \Delta(\alpha, \beta ; n), \beta-\alpha \neq \pi$, and $f(0) \neq \infty$. Then by Lemmas 7 and 8 , $f$ has no poles and zeros in $D$ except a simple zero at the origin. Furthermore, $f$ has no essential and algebraic singularities on $C$ except $n$ algebraic poles at $e^{i \alpha_{j}}$, $j=1,2, \ldots, n$, and $f$ is star-like in $D$ due to Lemmas 9 and 10 .

Finally, by applying Lemma 11 we see that the function $f$ can be represented by (14). In view of (12), we know that the boundary values $f\left(e^{i \theta}\right) \in R_{j}$, for all $\theta \in\left(\alpha_{j}, \alpha_{j+1}\right), j=1,2, \ldots, n$. This implies that the function $\gamma(t)$ defined in (14) is a 
step function with jump, say $\frac{1}{2} p_{j}$, where the associated number $p_{j} \pi$ denotes the angle between $R_{j}$ and $R_{j+1}$, due to the fact that

$$
\frac{1}{2} \sum_{j=1}^{n} p_{j}=\gamma(2 \pi)-\gamma(0)=1 \text { or } \sum_{j=1}^{n} p_{j} \pi=2 \pi \text {. }
$$

Substituting each jump into (14), we obtain

$$
f(z)=c z \prod_{j=1}^{n}\left(1-e^{-i \alpha_{j} z}\right)^{-p_{j}} \quad\left(c=f^{\prime}(0) \neq 0\right) .
$$

This gives (8) and the proof is complete.

9. Remarks and corollaries. In this last section, we shall give two remarks and two corollaries of Theorem 5. First, we note that the hypothesis $\beta-\alpha \neq \pi$ in Theorem 5 is necessary. This guarantees that $f(0)=0$ and therefore $f$ is star-like in $D$. Without this condition, there exist some univalent functions which cannot be represented by (8). To see this, we let $G$ be a simply connected domain bounded by $2 n(n>1)$ half rays which are formed pairwise symmetric with respect to the real axis and one of them lies on the real axis and passes through the origin. Then by the Riemann mapping theorem (see [10, Theorem 17.1]), there is a conformal mapping $f$ from $D$ onto $G$. Since the domain $G$ is symmetric with respect to the real axis, the function $f$ can be required to be symmetric on $D$, so that $f$ preserves the sector $\Delta(0, \pi)$ and, further, $f \in \Delta(0, \pi ; 2 n)$. Since $f(0) \neq 0, f$ cannot be represented by (8).

On the other hand, if $\beta-\alpha=\pi$ and $n$ is small, then by the same argument we can easily obtain the following result.

COROllary 1. If $f \in \Delta(\alpha, \beta ; n)$ with $\beta-\alpha=\pi$ and $n \leqslant 2$, and if $f$ is univalent in $D$, then

$$
f(z)=c z\left[\left(1-e^{-i \alpha_{1}} z\right)\left(1-e^{-i \alpha_{2}} z\right)\right]^{-1}+f(0), \text { for some } c \neq 0,
$$

where $\alpha_{1}=\alpha_{2}$ if and only if $n=1$.

In contrast to the function defined in (7), we have the following representation of a subclass in $\Delta(\alpha, \beta ; n)$.

COROllaRy 2. If $f \in \Delta(\alpha, \beta ; n)$, and $f$ is univalent in $D$, and if the set of all boundary values $f\left(e^{i \theta}\right)$ consists of $n$ half rays whose finite endpoints are all uniformly distributed on a circle, then

$$
f(z)=c z /\left(1-z^{n}\right)^{2 / n}, \text { for some } c \neq 0 .
$$

Finally, we remark that the hypothesis of univalency of $f$ is necessary in the representation (8). Without this restriction, there does exist a function $f$ in the class $\Delta(\alpha, \beta ; n)$ which is not univalent in $D$ and therefore cannot be represented by (8). For example, the function

$$
f(z)=f_{1}(z) \frac{1+z^{2}}{1-z^{2}}, \quad \text { where } f_{1}(z)=\frac{z}{1-z^{2}},
$$


is not univalent in $D$. By a simple computation, we have

$$
\operatorname{Im} f\left(r e^{i \theta}\right)=\frac{(\sin \theta) r\left(1-r^{2}\right)\left[\left(1+r^{2}\right)^{2}+4 r^{2} \cos ^{2} \theta\right]}{\left|1-r^{2} e^{i 2 \theta}\right|^{2}} .
$$

It follows that the function $f$ preserves the sector $\Delta(0, \pi)$ and has radial limits $f\left(e^{i \theta}\right)$ on the real line for every $\theta \neq 0, \pi$. Hence the function $f \in \Delta(0, \pi ; 2)$ and clearly $f$ cannot be represented by (8). Note that the associated function $f_{1}$ is univalent in $D$ and belongs to a different class $\Delta^{*}(0, \pi ; 2)$ by Corollary 2 , where the radial limits $f_{1}\left(e^{i \theta}\right)$ lie on the upper and lower imaginary axis, but $f_{1}$ preserves the same sector $\Delta(0, \pi)$.

\section{REFERENCES}

1. E. F. Collingwood and A. J. Lohwater, The theory of cluster sets, Cambridge Univ. Press, Cambridge, 1966.

2. J. L. Doob, The ranges of analytic functions, Ann. of Math. (2) 36 (1935), 117-126.

3. J. S. Hwang, On an extremal property of Doob's class, Trans. Amer. Math. Soc. 252 (1979), 393-398.

4. __ On the ranges of analytic functions, Trans. Amer. Math. Soc. 260 (1980), 623-629.

5. On a problem of Lohwater about the asymptotic behaviour of Nevanlinna's class, Proc. Amer. Math. Soc. 81 (1981), 538-540.

6. , On the Schwarz reflection principle, Trans. Amer. Math. Soc. 272 (1982), 711-719.

7. A. J. Lohwater, On the Schwarz reflection principle, Michigan Math. J. 2 (1953-1954), 151-156.

8. R. Nevanlinna and V. Paatero, Introduction to complex analysis, Addison-Wesley, Reading, Mass., 1969.

9. C. Pommerenke, Univalent functions, Studia Math., Göttingen, 1975.

10. W. Rogosinski, Über positive harmonische Entwicklungen und typischreelle Potenzreihen, Math. Z. 35 (1932), 93-121.

11. W. Seidel, On the cluster values of analytic functions, Trans. Amer. Math. Soc. 34 (1932), 1-21.

12. On the distribution of values of bounded analytic functions, Trans. Amer. Math. Soc. 36 (1934), 201-226.

Institute of Mathematics, ACademia Sinica, Taipei, Taiwan

Current address: Department of Mathematics, Michigan State University, East Lansing, Michigan 48824 